\title{
Microbiological profile of symptomatic and asymptomatic bacteriuria recovered from female patients with urinary tract infection
}

\author{
Una Jessica Sarker, Md. Sakil Munna and Saurab Kishore Munshi ${ }^{\ddagger}$ \\ Department of Microbiology, Stamford University Bangladesh, 51 Siddeswari Road, Dhaka \\ 1217, Bangladesh
}

Received 2 November 2013/Accepted 31 November 2013

\begin{abstract}
With the previous knowledge on the production of $\beta$-lactamase by the bacterial pathogens causing urinary tract infection, present study further investigated the presence of symptomatic and asymptomatic bacteriuria in female patients admitted into the Delta Medical College with suspected urinary tract infections (UTIs). The manifestation of uropathogens and their responses against locally available antibiotics (amoxicillin, $10 \mu \mathrm{g}$; cephradin, $30 \mu \mathrm{g}$; ciprofloxacin, $5 \mu \mathrm{g}$; cotrimoxazole, $23.8 \mu \mathrm{g}$; gentamicin, $10 \mu \mathrm{g}$; nalidixic acid, $30 \mu \mathrm{g}$ ) were inquired by means of conventional cultural techniques and double-disc diffusion methods, respectively. Among 110 urine samples collected from the patients with suspected UTI, 34 were found to be culture positive. Symptomatic $(64.7 \%)$ and asymptomatic $(\mathbf{3 6 . 3 \%})$ bacteriuria were noticed inside the puss cell of the UTI positive cases. Escherichia coli $(\mathbf{7 3 . 5 \%})$ was the dominant bacteria while Klebsiella spp. (26.5\%) was also exultant. Around $96 \%$ uropathogens were found to be sensitive against imipenem, and $75 \%$ against amikacin. $E$. coli was found to be sensitive against all of the antibiotics used, whereas Klebsiella spp. was found to be $100 \%$ resistant against nalidixic acid and cotrimoxazole.
\end{abstract}

Key words: Urinary tract infection; Pathogens; Antibiotic resistance; Symptomatic; Asymptomatic

Urinary tract infections (UTIs) are already known to be the most frequently encountered bacterial illness with a relatively higher frequency of onset among women (1-6). On an average of $50 \%$ of females manifest symptoms of UTI at certain stage during their lives because of their physiological pattern of anus and urethral meatus (6-10). The prevalence of infection also varies especially with the age and sex of the individual $(6,11,12)$.

The most frequent uropathogen is known to be the Gram negative bacterium Escherichia coli which have been reported to cause $80 \%$ of the community-acquired UTI and $40 \%$ of the healthcare-associated UTI $(6,13)$. Other common uropathogens include Klebsiella pneumoniae, Enterococcus spp., Pseudomonas aeruginosa, Candida spp., Proteus spp. and Enterobacter spp. (5, 6, 14, 15). The medication strategies of UTI have also been linked to the identification of the drug-resistance traits of the uropathogens against the commonly used antibiotics (5, 6, 10).

Recent studies conducted in Bangladesh also revealed that $12 \%$ of the rural women were suffering from asymptomatic bacteriuria (10). Our earlier investigation

${ }^{\ddagger}$ Corresponding Author: Mailing address. Saurab Kishore Munshi, Department of Microbiology, Stamford University Bangladesh, 51 Siddeswari Road, Dhaka 1217, Bangladesh; E-mail: noor.kishore016@yahoo.com. revealed the prevalence of uropathogens and their antibiotic resistance patterns as well as their extendedspectrum $\beta$-lactamase activity $(5,6)$. The current investigation further improvised the study of UTI in Bangladesh through (i) the manifestation of UTI among female patients admitted into the Delta Medical College based on their age and (ii) the responses of isolated uropathogens against locally available antibiotics commonly prescribed by the physicians.

\section{MATERIALS AND METHODS}

\footnotetext{
Study population. The study was conducted at Department of Microbiology, Delta medical college and Hospital. Patients who were prescribed by doctors for undergoing tests for UTIs were included in the study. A total of 110 randomly chosen female patients of different sex groups were included in this study (Table 1).

Ethical consideration. Ethical permission was not required in the study as the patients were prescribed by the doctors to undertake the UTIs test.

Isolation and identification of uropathogens. After collecting the specimens aseptically (16), physical and chemical examinations were carried out (data not shown), then samples were centrifuged and examined microscopically (Optima Biological Microscope G206, manufactured in Taiwan) (6).

For bacteriological analysis, Nutrient agar (NA), MacConkey agar (MAC) and Erosin Methylene Blue (EMB) agar plates were used. By means of a calibrated loop of $3 \mathrm{~mm}$ diameter, the appropriately labeled media plates were streaked aseptically with an aliquot of $0.01 \mathrm{ml}$ urine sample. The plates were then incubated aerobically at $37{ }^{\circ} \mathrm{C}$ for $12-24$ hours. Bacteriological identification was done by the examination of the overnight culture and Gram-staining. Standard biochemical tests were performed also to identify the bacteria of interest $(5,6,17)$.

Antibiotic susceptibility test. Agar disc diffusion assay was used to determine the bacterial susceptibility towards different antibiotics (amoxicillin, $10 \mu \mathrm{g}$; cephradin, $30 \mu \mathrm{g}$; ciprofloxacin, $5 \mu \mathrm{g}$; cotrimoxazole, $23.8 \mu \mathrm{g}$; gentamicin, $10 \mu \mathrm{g}$; nalidixic acid, $30 \mu \mathrm{g})$ in vitro as described previously $(5,6,18,19)$. The zone diameters for individual antimicrobial agents were translated into susceptible and resistant categories (20).
} 


\section{RESULTS AND DISCUSSION}

Prevalence of UTI according to age. Out of 110 patients, $34(30.9 \%)$ patients were found to bear UTI. Women in the age group 21-30 years were most likely to suffer from UTI (72.7\%) above than the age group of 10-20 years (Table 1). Noor et al. (6) reported most of the occurrence of UTI among 16-45 aged females. Such infections might occur through their shorter and closer urethra, which is indeed corroborating with the previous suggestive reports (6-10).

Microbiological profile of UTI. All the UTI positive samples were shaded with substantial number of total viable bacteria of $10^{5} \mathrm{cfu} / \mathrm{ml}$ or more (Table 2). Among $34,22(64.7 \%)$ UTI positive cases experienced symptomatic bacteriuria with recovery of 5-9 pus cells/high power field. Other 12 (35.3\%) UTI cases were found to have asymptomatic bacteriuria (Table 2). Notably, the presence of symptomatic and asymptomatic bacteriuria inside the pus cells (Table 3) accord the possibility of pyonephrosis (21).
TABLE 1. UTI positive cases among different patients' age group

\begin{tabular}{ccc}
\hline \multirow{2}{*}{ Age group } & \multicolumn{2}{c}{ Patients } \\
\cline { 2 - 3 } & Tested $(\mathrm{n})$ & UTI positive $(\%)$ \\
\hline 10-20 years & 22 & $4(11.8 \%)$ \\
21-30 years & 22 & $16(72.7 \%)$ \\
31-40 years & 22 & $8(36.4 \%)$ \\
41-50 years & 22 & $6(27.3 \%)$ \\
51-60 years & 22 & $0(0 \%)$ \\
\hline Total & 110 & $34(30.9 \%)$ \\
\hline
\end{tabular}

isolated in this study belonged to the Enterobacteriaceae family (Table 3). E. coli was identified as the most common causative agent of UTIs $(73.5 \%)$ over Klebsiella spp. $(26.5 \%)$, which was in consistence with the previous reports $(5,6)$.

Drugs resistance profile of the bacterial isolates causing UTI. The drug resistance and sensitivity profile of the microorganisms showed that $E$. coli isolates were sensitive to amoxicillin $10 \mu \mathrm{g}(80 \%)$; nalidixic acid $30 \mu \mathrm{g}$ (64\%), ciprofloxacin $5 \mu \mathrm{g}(24 \%)$; gentamicin $10 \mu \mathrm{g}$

TABLE 2. Number of viable bacteria and pus cells, and determination of symptomatic and asymptomatic bacteriuria in UTI positive samples

\begin{tabular}{|c|c|c|c|c|}
\hline $\begin{array}{c}\text { No. of UTI } \\
\text { positive sample }\end{array}$ & $\begin{array}{l}\text { Total viable bacteria } \\
(\mathrm{cfu} / \mathrm{ml})\end{array}$ & $\begin{array}{l}\text { Number of } \\
\text { pus cells } \\
\text { (cell/hpf) }\end{array}$ & $\begin{array}{c}\text { Symptomatic } \\
\text { bacteriuria } \\
(64.7 \%)\end{array}$ & $\begin{array}{c}\text { Asymptomatic } \\
\text { bacteriuria } \\
(35.3 \%)\end{array}$ \\
\hline 01 & $1.3 \times 10^{5}$ & $6-9$ & + & - \\
\hline 02 & $1.8 \times 10^{6}$ & $5-7$ & + & - \\
\hline 03 & $2.3 \times 10^{5}$ & $6-8$ & + & - \\
\hline 04 & $3.1 \times 10^{5}$ & $5-8$ & + & - \\
\hline 05 & $2.8 \times 10^{6}$ & $6-9$ & + & - \\
\hline 06 & $1.2 \times 10^{5}$ & $6-9$ & + & - \\
\hline 07 & $1.7 \times 10^{5}$ & $2-3$ & - & + \\
\hline 08 & $1.9 \times 10^{6}$ & $5-8$ & + & - \\
\hline 09 & $4.1 \times 10^{5}$ & $2-3$ & - & + \\
\hline 10 & $3.2 \times 10^{5}$ & $2-3$ & - & + \\
\hline 11 & $1.3 \times 10^{6}$ & $2-3$ & - & + \\
\hline 12 & $2.2 \times 10^{5}$ & $6-9$ & + & - \\
\hline 13 & $1.5 \times 10^{6}$ & $5-7$ & + & - \\
\hline 14 & $5.1 \times 10^{5}$ & $6-8$ & + & - \\
\hline 15 & $4.8 \times 10^{6}$ & $2-3$ & - & + \\
\hline 16 & $7.8 \times 10^{5}$ & $2-3$ & - & + \\
\hline 17 & $5.3 \times 10^{5}$ & $2-3$ & - & + \\
\hline 18 & $1.6 \times 10^{6}$ & $6-9$ & + & - \\
\hline 19 & $1.8 \times 10^{5}$ & $5-7$ & + & - \\
\hline 20 & $2.9 \times 10^{6}$ & $6-8$ & + & - \\
\hline 21 & $1.3 \times 10^{5}$ & $6-8$ & + & - \\
\hline 22 & $5.2 \times 10^{5}$ & $5-8$ & + & - \\
\hline 23 & $1.9 \times 10^{6}$ & $6-9$ & + & - \\
\hline 24 & $1.6 \times 10^{6}$ & $6-9$ & + & - \\
\hline 25 & $1.3 \times 10^{5}$ & $6-8$ & + & - \\
\hline 26 & $3.4 \times 10^{4}$ & $5-8$ & + & - \\
\hline 27 & $2.9 \times 10^{5}$ & $6-9$ & + & - \\
\hline 28 & $2.1 \times 10^{6}$ & $6-9$ & + & - \\
\hline 29 & $7.1 \times 10^{5}$ & $6-8$ & + & - \\
\hline 30 & $3.1 \times 10^{5}$ & $2-3$ & - & + \\
\hline 31 & $1.9 \times 10^{5}$ & $2-3$ & - & + \\
\hline 32 & $1.3 \times 10^{5}$ & $2-3$ & - & + \\
\hline 33 & $1.6 \times 10^{5}$ & $2-3$ & - & + \\
\hline 34 & $1.4 \times 10^{5}$ & $2-3$ & - & + \\
\hline
\end{tabular}

hpf = High power field; $(+)=$ Positive; $(-)=$ Negative 
TABLE 2. Identification and confirmation of the causative agents of urinary tract infections

\begin{tabular}{|c|c|c|c|c|c|c|c|}
\hline \multirow{2}{*}{ Isolate No. } & \multicolumn{3}{|c|}{ TSI } & \multirow{2}{*}{ Citrate } & \multirow{2}{*}{ Gram staining } & \multirow{2}{*}{$\begin{array}{c}\text { Colony } \\
\text { Characteristics on } \\
\text { EMB agar }\end{array}$} & \multirow{2}{*}{$\begin{array}{c}\text { Suspected } \\
\text { microorganisms }\end{array}$} \\
\hline & Slant & Butt & Gas & & & & \\
\hline 01 & $\mathrm{Y}$ & $\mathrm{Y}$ & + & + & Gram negative rods & Green Metallic sheen & E.coli \\
\hline 02 & $\mathrm{Y}$ & $\mathrm{Y}$ & + & - & Gram negative rods & Green Metallic sheen & E.coli \\
\hline 03 & $\mathrm{Y}$ & $\mathrm{Y}$ & + & + & Gram negative rods & Green Metallic sheen & E.coli \\
\hline 04 & $\mathrm{Y}$ & $\mathrm{Y}$ & + & + & Gram negative rods & Green Metallic Sheen & E.coli \\
\hline 05 & $\mathrm{Y}$ & $\mathrm{Y}$ & + & + & Gram negative rods & Glossy pink & Klebsiella spp. \\
\hline 06 & $\mathrm{Y}$ & $\mathrm{Y}$ & + & - & Gram negative rods & Green Metallic sheen & E.coli \\
\hline 07 & $\mathrm{Y}$ & $\mathrm{Y}$ & + & + & Gram negative rods & Green Metallic Sheen & E.coli \\
\hline 08 & $\mathrm{Y}$ & $\mathrm{Y}$ & + & + & Gram negative rods & Glossy pink & Klebsiella spp. \\
\hline 09 & $\mathrm{Y}$ & $\mathrm{Y}$ & + & + & Gram negative rods & Glossy pink & Klebsiella spp. \\
\hline 10 & $\mathrm{Y}$ & $\mathrm{Y}$ & + & + & Gram negative rods & Green Metallic Sheen & E. coli \\
\hline 11 & $\mathrm{Y}$ & $\mathrm{Y}$ & + & + & Gram negative rods & Green Metallic Sheen & E. coli \\
\hline 12 & $\mathrm{Y}$ & $\mathrm{Y}$ & + & + & Gram negative rods & Glossy pink & Klebsiella spp. \\
\hline 13 & $\mathrm{Y}$ & $\mathrm{Y}$ & + & + & Gram negative rods & Glossy pink & Klebsiella spp. \\
\hline 14 & $\mathrm{Y}$ & $\mathrm{Y}$ & + & + & Gram negative rods & Glossy pink & Klebsiella spp. \\
\hline 15 & $\mathrm{Y}$ & $\mathrm{Y}$ & + & + & Gram negative rods & Green Metallic Sheen & E. coli \\
\hline 16 & $\mathrm{Y}$ & $\mathrm{Y}$ & + & + & Gram negative rods & Green Metallic Sheen & E. coli \\
\hline 17 & $\mathrm{Y}$ & $\mathrm{Y}$ & + & + & Gram negative rods & Green Metallic Sheen & E. coli \\
\hline 18 & $\mathrm{Y}$ & $\mathrm{Y}$ & + & + & Gram negative rods & Green Metallic Sheen & E. coli \\
\hline 19 & $\mathrm{Y}$ & $\mathrm{Y}$ & + & + & Gram negative rods & Green Metallic Sheen & E. coli \\
\hline 20 & $\mathrm{Y}$ & $\mathrm{Y}$ & + & + & Gram negative rods & Green Metallic Sheen & E. coli \\
\hline 21 & $\mathrm{Y}$ & $\mathrm{Y}$ & + & + & Gram negative rods & Green Metallic Sheen & E. coli \\
\hline 22 & $\mathrm{Y}$ & $\mathrm{Y}$ & + & + & Gram negative rods & Glossy pink & Klebsiella spp. \\
\hline 23 & $\mathrm{Y}$ & $\mathrm{Y}$ & + & + & Gram negative rods & Glossy pink & Klebsiella spp. \\
\hline 24 & $\mathrm{Y}$ & $\mathrm{Y}$ & + & + & Gram negative rods & Glossy pink & Klebsiella spp. \\
\hline 25 & $\mathrm{Y}$ & $\mathrm{Y}$ & + & + & Gram negative rods & Green Metallic Sheen & E. coli \\
\hline 26 & $\mathrm{Y}$ & $\mathrm{Y}$ & + & + & Gram negative rods & Green Metallic Sheen & E. coli \\
\hline 27 & $\mathrm{Y}$ & $\mathrm{Y}$ & + & + & Gram negative rods & Green Metallic Sheen & E. coli \\
\hline 28 & $\mathrm{Y}$ & $\mathrm{Y}$ & + & + & Gram negative rods & Green Metallic Sheen & E. coli \\
\hline 29 & $\mathrm{Y}$ & $\mathrm{Y}$ & + & + & Gram negative rods & Green Metallic Sheen & E. coli \\
\hline 30 & $\mathrm{Y}$ & $\mathrm{Y}$ & + & + & Gram negative rods & Green Metallic Sheen & E. coli \\
\hline 31 & $\mathrm{Y}$ & Y & + & + & Gram negative rods & Green Metallic Sheen & E. coli \\
\hline 32 & $\mathrm{Y}$ & $\mathrm{Y}$ & + & + & Gram negative rods & Green Metallic Sheen & E. coli \\
\hline 33 & $\mathrm{Y}$ & $\mathrm{Y}$ & + & + & Gram negative rods & Green Metallic Sheen & E. coli \\
\hline 34 & $\mathrm{Y}$ & $\mathrm{Y}$ & + & + & Gram negative rods & Green Metallic Sheen & E. coli \\
\hline
\end{tabular}

$\mathrm{TSI}=$ triple sugar iron test; $\mathrm{Y}=$ yellow (acidic); $\mathrm{R}=$ red (alkaline); EMB = Erosin Methylene blue
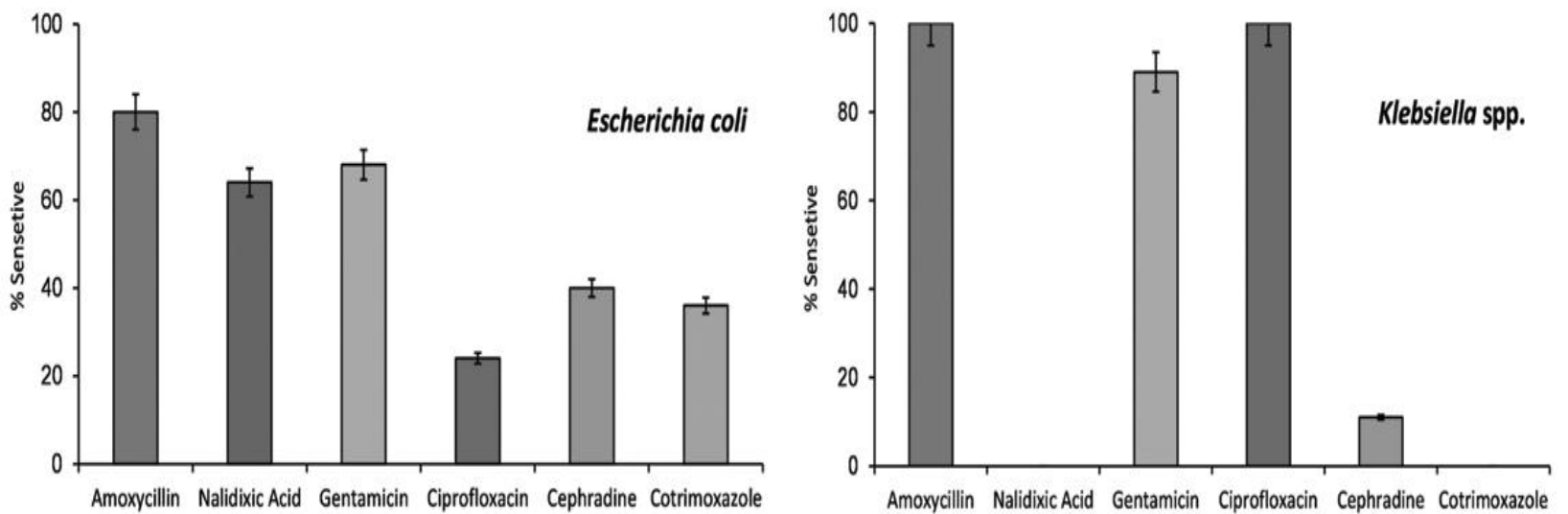

FIG. 1. Antimicrobial resistance pattern of E. coli, Klebsiella spp. against amoxycillin (10 $\mu \mathrm{g})$, nalidixic acid $(30 \mu \mathrm{g})$, ciprofloxacin $(5 \mu \mathrm{g})$, gentamicin $(10 \mu \mathrm{g})$, cephradin $(30 \mu \mathrm{g})$, cotrimoxazole $(23.8 \mu \mathrm{g})$. E. coli was found to be sensitive to some extent against all of the antibiotics used. Notably, Klebsiella spp was found to be $100 \%$ resistant against nalidixic acid and cotrimoxazole. The presented data were statistically analyzed by showing standard errors considered as 5\%. All experiments were carried out three times and $95 \%$ accuracy was found. 
cephradin $30 \mu \mathrm{g}$ (40\%); cotrimoxazole $23.8 \mu \mathrm{g}$ (36\%) (Figure 1), whereas Klebsiella spp. were found to be $100 \%$ resistant against nalidixic acid $30 \mu \mathrm{g}$; and cotrimoxazole $23.75 \mu \mathrm{g}$ (Figure 1). Considerably, several studies in the developed and developing countries have revealed higher levels of resistance against the most commonly used antibiotic agents, possibly due to the dissemination of the drug-resistance genes within the infectious microorganisms $(6,18,22-$ 25).

\section{CONCLUSION}

Overall, the current investigation clearly showed the incidences of urinary tract infections are most frequent in female patients within the age group of 21-30 years admitted into the Delta Medical College. Moreover, encountering asymptomatic bacteriuria in pus cells of the tested population pictured severe health risk due to its unpredictable nature and appeal more attention from the public health sectors.

\section{ACKNOWLEDGEMENTS}

Authors thank Department of Microbiology of Delta medical college and Hospital for providing the facilities for all the experiments described here.

\section{REFERENCES}

1. Travis, L. B., and B. H. Bruhard. 1996. Infections of the urinary tract, p. 21-28. In A. M. Rudolph (ed.), Rudolph's pediatrics. Appleton \& Lange, Connecticut.

2. Allasio, D., and H. Fischer. 2005. Maintaining a sterile urinary tract: the role of antimicrobial peptides. J. Urol. 182: 21-28.

3. Parveen, K., A. Momen, A. A. Begum, and M. Begum. 2011. Prevalence of urinary tract infection during pregnancy. J. Dhaka National Med. Coll. Hos. 17: 8-12.

4. Demilie, T., G. Beyene, S. Melaku, and W. Tsegaye. 2012. Urinary bacterial profile and antibiotic susceptibility pattern among pregnant women in north-west Ethiopia. Ethiop. J. Health Sci. 22: 121-128.

5. Khan, S. A., F. Feroz, and R. Noor. 2013. Study of extended-spectrum $\beta$ lactamase-producing bacteria from urinary tract infections in Bangladesh. Tzu Chi Med. J. 25: 39-42.
6. Noor, A. F., et al. 2013. Prevalence and antibiogram profile of uropathogens isolated from hospital and community patients with urinary tract infections in Dhaka city. J. Bangladesh Acad. of Sci. 37: 57-63.

7. Kunin, C. M. 1997. Urinary tract infections in adults, p. 128-164. In C. M. Kunin (ed.), Urinary Tract Infections, $5^{\text {th }}$ ed. Williams \& Wilkins, Baltimore.

8. Pezzlo, M. 1988. Detection of Urinary Tract Infections by Rapid Methods. Clinical Microbiol. Rev. 1: 268-280

9. Wilson, A. P., et al. 1986. Prevalence of urinary tract infection in homosexual and heterosexual men. Genitourin. Med. 62: 189-190.

10. Ullah, M. A., A. Barman, M. A. Siddique, and A. K. M. E. Haque. 2007. Prevalence of asymptomatic bacteriuria and its consequences in pregnancy in a rural community of Bangladesh. Bangladesh Med. Res. Counc. Bull. 33: 6064.

11. Klevan, J. L., and A. De Jong. 1990. Urinary tract symptoms and urinary tract infection following sexual abuse. Am. J. Dis. Child. 144: 242-244.

12. Griebling, T. L. 2001. Urinary Tract Infection in Women, p. 587. In M. S Litwin, and C. S. Saigal (ed.), Urologic diseases in America. US Government Printing Office, Washington, DC.

13. Ronald, A. 2003. The etiology of urinary tract infection: traditional and emerging pathogens. Dis. Mon. 49: 71e82.

14. Tolkoff, N. E., and R. H. Rubin. 1986. Urinary tract infection: significance and management. Bull. NY Acad. Med. 62:131e48.

15. Goering, R. V., et al. 2008. Mims' medical microbiology, $4^{\text {th }}$ ed. Mosby, Philadelphia.

16. Skobe, C. 2004. The Basics of specimen collection and handling of urine testing, LabNotes 14: 2-7.

17. Cappuccino, J. G., and N. Sherman. 1996. Microbiology- A Laboratory Manual. $4^{\text {th }}$ ed. The Benjamin/Cummings Publishing Co., Inc., Menlo Park, California.

18. Dutta, S., et al. 2013. Study of antimicrobial susceptibility of clinically significant microorganisms isolated from selected areas of Dhaka, Bangladesh. Bangladesh J. Med. Sci. 12: 34-42.

19. Bauer, A. W., W. M. M. Kirby, J. C. Sherris, and M. Tierch. 1966 Antibiotic susceptibility testing by a standardized single disc method. Am. J. Clin. Pathol. 45: 493-496.

20. Ferraro, M. J., W. A. Craig, and M. N. Dudley. 2001. Performance standards for antimicrobial susceptibility testing - NCCLS informational supplement, $11^{\text {th }}$ ed. NCCLS, Pennsylvania, USA.

21. Najar, M. S., C. L. Saldanha, and K. A. Banday. 2009. Approach to urinary tract infections. Indian J. Nephrol. 19: 129-139.

22. Gul, N., T. Y. Mujahid, and S. Ahmad. 2004. Isolation identification and antibiotic resistance profile of indigenous bacterial isolates from urinary tract infection patients. Pak. J. Biol. Sci. 7: 2051e4.

23. Alos, J. I., M. G. Serrano, J. L. Gomez-Garces, and J. Perianes. 2005 Antibiotic resistance of Escherichia coli from community-acquired urinary tract infections in relation to demographic and clinical data. Clin. Microbiol. Infect. 11: 199-203.

24. Kothari, A., and V. Sagar. 2008. Antibiotic resistance in pathogens causing communityacquired urinary tract infections in India: A multicenter study. J. Infect. Dev. Ctries. 2:354e8.

25. Olukemi, O. A., and F. Adeola. 2012. Extended-spectrum beta-lactamase production among clinical isolates of Escherichia coli. Int. Res. J. Microbiol. 3: $140 \mathrm{e} 3$. 